\title{
Synonymy between Token-Reflexive Expressions
}

\begin{abstract}
Synonymy, at its most basic, is sameness of meaning. A token-reflexive expression is an expression whose meaning assigns a referent to its tokens by relating each particular token of that particular expression to its referent. In doing so, the formulation of its meaning mentions the particular expression whose meaning it is. This seems to entail that no two token-reflexive expressions are synonymous, which would constitute a strong objection against token-reflexive semantics. In this paper, I propose and defend a notion of synonymy for token-reflexive expressions that allows such expressions to be synonymous, while being a fairly conservative extension of the customary notion of synonymy.
\end{abstract}

\section{The Problem}

Suppose that some expressions are token-reflexive, that is, their meaning assigns an object as a referent to each token (possibly relative to a parameter, like a context) by somehow relating that object to that very token. For instance, a theory could make the meaning of the first person pronoun "I" to be token-reflexive by saying that its meaning assigns the speaker / user of each particular token of "I" as a referent of that token. It is not important here exactly what tokens are, or how to specify the meanings. What matters is the reflexivity: the view that the formulations of the meanings of some expressions ineliminably mention those very expressions.

Now suppose that we take synonymy to be sameness of meaning. We immediately run into a problem: since the meaning rules of each particular token-reflexive expression mention that very 
expression, no two different token-reflexive expressions can be synonymous. My goal here is to give an account of synonymy for such expressions that avoids this bad consequence.

In order for this to count as a real problem, I need to make some assumptions. First, one must allow some role for synonymy. If it is a notion that looks suspicious from the beginning, this problem does not arise.

Second, it would be best if the reader allowed that some expressions are token-reflexive. In one sense, this is trivially true: such expressions can always be introduced by definition (and I will do some of that). But the problem is more interesting if there are natural language expressions with this property. I believe that demonstratives ("this", "she", etc.) are token-reflexive, though I will not argue for this here. Briefly, my reason is that I believe that the speaker's intentions play a role in securing a referent for each demonstrative token, and that these intentions are ineliminably aimed at that particular token of that particular expression. ${ }^{1}$ If other context-sensitive expressions work like demonstratives, then they too are token-reflexive.

If the reader is not inclined to find it plausible that there are token-reflexive expressions in natural languages, they may read this paper as an argument that the alleged lack of synonymy is not a good reason to discount the view that there are token-reflexive expressions.

Third, one needs to start out thinking of meanings as offered by popular theories of meaning (I will give some examples in §2.2). By the end of the paper, I will propose a notion of meaningform, which I would hope is natural enough. If the reader dislikes the theories I am relying on, they may read the paper as an argument against those theories, although I suspect that similar problems arise for any theory fine grained enough to be applicable to token-reflexive expressions.

A more theoretical way to describe interest in the problem stems from the observation that the expressions tout court are not the right kind of vehicle for reference, since there are expressions, like context-sensitive ones, whose referent differs depending on various factors, without leading to

\footnotetext{
${ }^{1}$ Kaplan (1989) famously argued against token-reflexivity, both in its Reichenbachian version, and more generally against any such theory; but in Kaplan (1999) he seems to have changed his mind about the latter. I do not mean this only as an appeal to authority; rather, I am using this example to try to convince the reader that this is not a crazy idea. I am also explicitly not arguing against Kaplan's classic view; I am talking about another kind of view, and addressing one possible problem it might have.
} 
a charge of ambiguity. Furthermore, their reference is not a job for pre-semantics, since they have a stable meaning that is characterizable in semantics proper. Expression semantics can be rescued by relativizing reference to indices, or contexts, or some such way to represent the factors in question. This led Kaplan (1989) to argue that "I" ought to get a semantic value even when its referent is sleeping, and thus in no way an agent or a speaker of any expression whatsoever. I would argue that this gives too much power to the semanticist. Assigning referents may be the business of the speaker, of the word, of the world, of the situation, but the semanticist ought to be an observer and a describer, not an assigner. To escape this autocracy of the semanticist, one may turn to tokens, or uses, or utterances, and claim that, by their place in the world, they come equipped with referents before the semanticist gets to look at them. So I am the referent of my uses of "I" because I am the user of those uses, not because I am so assigned by a semanticist. This gives a central role to tokens, and this role plausibly gets reflected in the meanings of such expressions.

Let me propose a particular way to formulate our problem. Suppose that, in addition to the meaning rule proposed for "I" above, we propose something similar for its French counterpart, "je": its meaning assigns the speaker / user of each particular token of "je" as a referent of that token. Since this rule mentions "je", it is a different rule from the one for "I", which mentions the English word, rather than the French one. This seems to entail that "I" and "je" are not synonymous. But if any two expressions are good candidates for synonymy, these are surely high up on the list. So we have an inconsistent triad:

(A) The formulation of the meaning of each token-reflexive expression mentions that very expression.

(B) Synonymy is sameness of meaning.

(C) There are synonymous token-reflexive expressions. ${ }^{2}$

\footnotetext{
${ }^{2}$ The story of this puzzle is somewhat complicated. The formulation is my own, but the puzzle itself was first noted independently by Braun (forthcoming) (written in 1998-1999) and Zimmermann (1997), the latter being mostly a paper about a related, but different problem discussed in Tsohatzidis (1992), which is about the apparent need to distinguish semantically between the French pronouns "tu" and "vous", both second person singular, with the former being informal, and the latter formal. I believe that the solution to that problem is also to be found by thinking about token-reflexivity, but I will not address that issue here.
} 
In this paper, I will propose a modified version of (B), so that the inconsistency goes away. The basic idea is this: although "I" and "je" have different meanings in the strictest sense of the word, they play the same role in language, namely they both express the first person pronoun, and it is in this respect that they are synonymous. Along the way, I will argue against views that reject (A) or (C), as well as against other ways of rejecting (B). The arguments will sometimes be very short (such as the one against views rejecting (C) in $§ 6.1$ ), and other times quite technical (such as the one against a view rejecting (A) in $§ 6.2$ ), but at all times I will be guided by the intuitive and for-now-inchoate idea that there is a useful notion of synonymy as playing the same role in a language.

In the next section, I make some of the claims in $(A)-(C)$ more precise, before moving on to stating my preferred solution to the problem.

\section{Some Preliminary Remarks}

\subsection{Which Expressions Are Token-Reflexive?}

Which expressions, if any, are token-reflexive is a matter of some dispute. Reichenbach (1947) argued that indexicals, like "I" and "today" are token-reflexive. Perry $(2001,1997)$ went even further, and argued that they are not merely token-reflexive, but utterance-reflexive, since one token can be used repeatedly, to make different utterances, with varying semantic values (imagine a sign bearing a token of the word "I" being used by different people at different times to say something about themselves). Kaplan (1989) argued against Reichenbach's views specifically, but more broadly argued that there can be no logic of utterances, and thus that there is no good semantics for them either.

I will assume that Kaplan's worries can be addressed, and that indexicals and demonstratives are at least token-reflexive (if they are utterance-reflexive, the puzzle is even more pressing, assuming that there are no token-less utterances). One influential response to Kaplan is offered in 
García-Carpintero (1998), and I am in broad agreement with it. ${ }^{3}$ The distinction between tokenreflexive views and utterance-reflexive views is very important, and not trivial to make (see Kaplan (1999); Braun (forthcoming) for more discussion, both of which contain ideas about how such semantics could go). The issue, is, however, orthogonal to this paper: my proposal for tokenreflexive synonymy can easily be adapted to utterance-reflexive synonymy, since the main obstacle to synonymy is the fact that the expression is mentioned in the meaning rule, and not exactly in what sense the meaning rule is reflexive. This is a paper about the way thinking about reflexivity affects our understanding of synonymy, independently of the detailed features of the mechanism of reflexivity.

\subsection{The Problem Is Fairly Theory-Neutral}

There are two ways in which the synonymy problem is theory-neutral. The first may be invisible, given how I set up the problem: I assumed that tokens of expressions refer. Some philosophers have argued that this kind of view is based on a confused blending between two perfectly fine notions, namely speaker reference and expression reference (which may be relative to a context). ${ }^{4}$ The synonymy problem arises for these theories too, so long as they hold that speaker meaning is constrained by the standing meaning of expressions, and, crucially, if there are expressions whose standing meaning is, somehow, reflexive. Given these fairly plausible assumptions, these views would similarly entail the problematic claim that such expressions would never have identical meanings.

Secondly, if one assumes that tokens (or utterances, or uses, etc.) refer, there still may be wide disagreement about how to specify meanings. Thesis (A) is therefore most likely to vary in its content depending on one's theory of meaning. But the problem is quite independent of any particular theory of meaning. Below, I offer a list of possible formulations of the meaning of "I" in different frameworks. In each of these I intend "every token" to pick out every possible token.

\footnotetext{
${ }^{3}$ However, see Predelli $(1998,2006,2004)$ for a defense of Kaplan's misgivings, along with many new arguments against token-reflexive semantics.

${ }^{4}$ For example, Bach (1992), Schiffer (2003, p. 112) and Neale (2016, §12.11).
} 
Throughout the paper, I will drop the modal for ease of exposition. ${ }^{5}$

Character Rule: The meaning of "I" is the following rule: every token of "I" refers to the speaker of that token of "I". 6

Character Function: The meaning of "I" is the function from each token of "I" to the speaker of that token of "I".

Context Function: The meaning of "I" is the function from each context in which "I" is tokened to the speaker in that context. ${ }^{8}$

Truth-Conditional Meaning: Any token of an English sentence of the form $\phi(\mathrm{I})$ is true just in case $\phi(x)$ is true relative to an assignment that assigns the speaker of that token of "I" to $\chi .{ }^{9}$

Each of these formulations essentially mentions the expression itself, and thus each such rule is different from the corresponding rule for "je". Take, for example, the Context Function rule. The problem here, which is easily adaptable to the other formulations, is that there are contexts in which "I" is tokened, but "je" is not; and thus the two functions are different. Or take the Character Rule formulation: the rule for "I" is about tokens of "I", and thus says nothing about tokens of “je”, nor vice-versa.

One might be tempted to propose a hybrid rule as the meaning of both "I" and "je", such as:

Composite Character Rule: Each token of "I" or "je" refers to the speaker of that token of "I" or "je".

\footnotetext{
${ }^{5}$ These are only simplified versions, of course; truth might be relative to a context, or a world, or a time; assignments might be needed, interpretations, etc. I am focusing on the parts of such rules that are most relevant to our discussion.

${ }^{6}$ This is a simplified version of the non-formal view in Kaplan (1989). It is customary to say that Kaplanian characters are just functions (see the next notion of meaning); see, for instance, Neale (2016, p. 294). I prefer a reading according to which the formal notion is merely a representation of the intuitive, non-technical idea.

${ }^{7}$ The source of this formulation is Kaplan (1989), although he thinks of this as a mere way to represent the meaning of "I", rather than being its meaning; see Braun (1995) for further discussion.

${ }^{8}$ The source of this formulation is Braun (forthcoming).

${ }^{9}$ See Zimmermann (1997, p. 147) for a variant of this formulation.
} 
The problem with this rule is that the meaning of an expression must be something that speakers of that language know in virtue of being competent with that word. And yet it is implausible to claim that the fundamental notion here is not competence with each expression in turn, but rather competence with both expressions at the same time. Rather, it should be the other way round: a speaker of both languages does indeed know something like the Composite Character Rule in virtue of knowing the Character Rule of each expression in turn. Besides, the composite rule really should address any realization of the first person pronoun in any possible language, and that is surely not something speakers can be expected to know.

\subsection{Who Is This a Problem for?}

The puzzle is pressing for two kinds of views, which are separable, though not necessarily separated.

First, one may think that reference is only a property of tokens, or uses, or utterances, rather than a property of expression types. One may hold this view because one thinks, along with Strawson (1950), Perry (2001) that people refer by using an expression, and that expressions themselves don't refer. In this case, the notion of the referent of a token may be derived from the fundamental notion of a person referring: a token of an expression refers to $x$ iff the person using that token is thereby referring to $x$. For our purposes, it does not matter which notion one thinks of as fundamental. What matters is that this line of thought may lead one to say that there is no such thing as, or that there is something wrong with the idea of, reference in the absence of a token, and, in particular, that there is no such thing as a referent of an expression type.

Suppose that one holds such a view. Then, plausibly, we would get the result that "pail" and "bucket" have different meanings, just like "I" and "je" above:

Character Function for "pail": The meaning of "pail" is the function from each token of "pail" to the property of being a pail/bucket.

Character Function for "bucket": The meaning of "bucket" is the function from each token of 
"bucket" to the property of being a pail/bucket.

The last part of these is unproblematic, assuming, as I would, that the property of being a bucket is the same as the property of being a pail; or one may instead forgo talk of properties, and talk about the class of pails in that world, etc. The problem comes from the fact that the two functions have distinct domains, namely the class of "pail"-tokens and the class of "bucket"-tokens.

I take it that this result is unacceptable: it would entail that there are no synonyms among expressions for whom referents are only assigned to tokens. Under this guise, the puzzle threatens a particular kind of semantic theory, rather than prototypical token-reflexive expressions like "I" and "je", namely a theory that thinks of reference as requiring a token, and thus of meaning as ascribing referents only to tokens. ${ }^{10}$

The second way one may run into trouble with the puzzle is if one thinks for some other reason that (C) above is true, i.e. that there are token-reflexive expressions. Such a semanticist would find coherent and unproblematic the notion of meanings that assign referents to expression types. But they may think that some expressions simply have a different kind of meaning, which only assigns a referent to tokens. My favorite example are demonstratives. Suppose, as I think, that a demonstratives cannot refer to $x$ in the absence of a speaker's intention to refer to $x .{ }^{11}$ I take this to lead one naturally to thinking that only tokens of demonstratives can refer, since speakers' intentions connect tokens to referents, rather than types. This would be reflected in the meanings of demonstratives, which would only talk of tokens referring, rather than types.

\section{Synonymy in the Purest Case of Token-Reflexivity}

Before we get to "I" and "je", let me introduce some expressions whose meaning I get to fix, which will allow me to focus solely on token-reflexivity. Consider, then, the purest form of token-

\footnotetext{
${ }^{10}$ I thank David Braun for pointing out the consequences of this view for expressions that one would not think of as paradigmatically token-reflexive.

${ }^{11}$ King (2014) claims that this is the majority view. See also Radulescu (2017).
} 
reflexivity, namely two expressions whose tokens always refer to themselves and nothing else: ${ }^{12}$

- Every token of $\zeta$ refers to that token of $\zeta^{13}$

- Every token of $\eta$ refers to that token of $\eta$.

My first claim is that $\zeta$ and $\eta$ are synonymous. I have two arguments for this claim. First, if they belonged to different languages, I would think it correct to offer one as the translation of the other. Translation is always a difficult matter, and even more so when we consider token-reflexive expressions. As Burge (1978, p. 137) puts it, "translation preserves self-reference if and only if it does not preserve reference”. Before addressing $\zeta$ and $\eta$, consider a point made in Hart (1970, pp. 523-524), namely that both (2) and (3) can be good translations of (1):

(1) This sentence is in English.

(2) Dieser Satz ist auf english.

(3) Dieser Satz ist auf deutsch.

A German teacher may expect their students to say that (2) is the correct translation. Note that this translation does not give us sameness of reference, nor of truth value, but each expression matches its default translation, i.e. what I would call its synonym. But if one wants to preserve the truth value and the self-referentiality of (1), e.g. if one were translating a philosophy paper, (3) would be better, though clearly "deutsch" is not the canonical translation of "English". As Burge (1978) notes in his discussion of similar sentences, (3) also better captures the speaker's intention and the overall point of asserting (1). Note that in both cases, "this sentence" is translated as "dieser Satz", and that complications arise only for the rest of the sentence. Neither Hart nor

\footnotetext{
${ }^{12}$ These are simplified versions of $\Theta^{*}$ as introduced in Reichenbach (1947, p. 287). Expressed as a character rule, the meaning of Reichenbach's $\Theta^{*}$ is the rule that says that each token refers to the largest expression token of which it is a part (e.g. a sentence).

${ }^{13}$ In the metalanguage, small Greek letters are names of the expression type, not of its tokens. In the language under consideration, the expression type does not have a name, and tokens of the small Greek letters always name themselves.
} 
Burge talk about synonymy. But if translation is a guide to synonymy, then "this sentence" and “dieser Satz" deserve to count as synonymous.

Keeping in mind the desideratum of maintaining self-reference, consider again $\zeta$ and $\eta$. I do not claim here that one is the only possible translation of the other. Perhaps in some translations one would need to introduce a new expression, maybe even the one in the other language; or a composite expression, performing the same role. Translation typically has many goals. For instance, in a meeting between an English speaker and a German speaker where each is joined by a translator, (4) might be what is expected of the translator, maybe because what matters is what each sentence says, rather than preserving self-referentiality:

(4) (1) ist auf english.

But any deviation from literal translation needs to have some justification; we saw one such justification in the English / German example above. But I would still contend that synonymy is the standard for default translation, a standard that is often superseded by other goals, but still the default one. To test your intuitions here, think not of Greek letters, whose artificiality is worn on their sleeves, but of something like English with the addition of "this-expression", used only to refer to that token of that expression in English ${ }^{+}$. Now move to French ${ }^{+}$, which is just like French, but we postulate that "cette-expression" can only be used in a manner similar to "this-expression". Since in French "cette-expression" can be used so that its tokens refer to themselves, French" is just French with the restriction that that expression is to be used only that way. I would translate one expression with the other, unless some reason would compel me to do otherwise.

The second argument is this: having learned one of the expressions, you only need to be told that the other is just like it, mutatis mutandis. In other words, a speaker of a language that contained $\zeta$, wanting to learn how to say $\zeta$ in the new language, would just need to be told that they should use (tokens of) $\eta$. This argument is somewhat independent of claims about translation, in that even if one were unsure about translation practices, which might incorporate complicated norms that may have to do with considerations independent of meanings, one could still be moved by considerations about learning a new language. 
Admittedly, synonymy in this realm has odd features. For instance, synonymy does not entail coreference. This is troubling, since if one could show that there is a bucket which is not a pail, one would thereby be naturally taken to have shown that "bucket" and "pail" are not synonyms. There is a choice to be made here. One option is to insist that coreference is a sine qua non of synonymy. Then we easily get the conclusion that there is no synonymy for expressions like $\zeta$. The second option is to rethink this condition in light of thinking about sameness of meaning in the token-referential area.

If this were merely a debate about what gets to be called "synonymy", I see no reason to prefer one option over the other, except possibly for the weight of tradition (but tradition for labels is a fragile thing, and all changes of tradition start out being anti-tradition).

I do not think, though, that this is just a dispute about labels. So let me flesh out the objection here. We could start by focusing on "bucket" and "pail". Suppose that they do have the same meaning. One might say that it is a consequence of their having the same meaning that they are (necessarily, analytically) coreferential. Perhaps one thinks this is because meaning determines reference, and if two expressions have the same meaning, then they are guaranteed to have the same referent. But for context sensitive expressions, meaning does not determine reference on its own; rather, reference is determined relative to a context. So now the original picture needs to be a bit more complicated. Suppose that "I" and "je" are synonymous. Then, one might think, their meanings, which, we are assuming, are identical, determine the same referent relative to each context.

If "I" and "je" are token-reflexive, as I have been assuming, this picture does not work, since, by hypothesis, there are contexts in which one is tokened and the other is not, so relative to some contexts, one gets a referent, and the other does not.

As always, we have several options. One would be to say that this shows that "I" and "je" are not token reflexive. In this paper, I am assuming this option away. The second option would be to say that this shows that token reflexives are never synonymous. The third option, to be discussed in $§ 6.2$, would have us modify the meanings of "I" and "je", so that they do come out synonymous 
and coreferential relative to all tokens. The fourth option, which I find more interesting, is to stop for a moment and rethink what follows from claiming that two expressions are synonymous. This allows us to think about expressions in an area of the language that was not under focus when talk of synonymy as entailing coreference was being converted into tradition. If I am right about the genealogy of our notion of synonymy, the proper way to think about its application to tokenreflexivity is not to assume that everything will be as we expect it to be, but rather to be open to changing what needs changing when one moves from one kind of expression to another.

But before we do that, let me mention a distinct problem for my view, one that I find even more unsettling (before we think about it the right way, of course). The starkest contrast between buckets and pails on the one hand, and token-reflexives on the other, is that all the tokens of this sentence are true:

(5) $\zeta \neq \eta$

This looks like a problem for anyone claiming that $\zeta$ and $\eta$ are synonyms. Then again, the following sentence also has only true tokens:

(6) $\zeta \neq \zeta$

(6) contains just one expression, and the truth of all its tokens does not show that $\zeta$ is ambiguous. Rather, it just highlights the token-reflexivity of that expression. Similarly, the guaranteed truth of all tokens of (5) does not show that $\zeta$ and $\eta$ differ in meaning. Synonymy here, I claim, is weaker than the standard notion.

So is there a sense in which $\zeta$ and $\eta$ are synonymous? Clearly, not in that their meanings are identical, if their meaning is given in any of the ways sketched above. But their meanings do have something in common, namely they are both instances of what I will call a "meaning-form":

(7) Every token of $\mathrm{T}$ refers to that token of $\mathrm{T}$.

My claim, then, is that synonymy is not sameness of meaning, but rather sameness of meaningform. Meaning-forms are just like plain old meanings, in any of the formulations above, but 
abstracting away from the expression in question (and the language, if we are looking for crosslinguistic synonymy). So knowing the meaning of $\zeta$ is to know one instance of (7), the meaningrule for the purest token-reflexive expressions. And knowing the meaning of its synonym, $\eta$, is to know a different instance of the same meaning-form.

In terms of our inconsistent triad, I propose to replace $(B)$ with $\left(\mathrm{B}^{*}\right)$ :

$\mathrm{B}^{*}$ Synonymy is sameness of meaning-form.

The replacement will make no change to the usual notion of synonymy, as long as it is only applied to expressions whose meaning rules do not mention those very expressions, since the process of abstraction from the expression itself leaves those meanings intact. So whatever the meaning of "bucket" is, so long as it is not token-reflexive, it is identical to its meaning-form. The difference only becomes important once reflexivity comes on the stage.

A reviewer worries that this proposal makes token-reflexive expressions sui generis, and that this would lower our credence in the existence of token-reflexive expressions. I think exactly the opposite. As we saw above, the translation of token-reflexive terms usually does not preserve reference. So we should expect token-reflexive expressions to need some special treatment. I propose no change to the notion of meaning for their sake. I only propose a modification of the notion of synonymy, in such a way that we get the usual results for the typical expressions we think of when we think about synonymy, but also the correct result for token-reflexive expressions. In fact, I would argue that the new notion of synonymy should raise our credence in the existence of token-referential expressions.

When are meaning-forms identical? The answer cannot be give in purely extensional terms. Note, for instance, that (7) yields the same results as the following, obviously distinct, rule:

(8) Every token of $\mathrm{T}$ refers to that token of $\mathrm{T}$ just in case $2+2=4$, and to the Moon otherwise.

Though I admit that this is an important issue, it is not new to this framework. Any extensional characterization of synonymy is likely to run into the same issue. Since I see no reason to think that sameness of meanings must be defined extensionally, I will leave this problem aside. 
Before I consider some objections, let me show how this idea can be applied to "I" and "je".

\section{Synonymy between First Person Singular Pronouns}

$\zeta$ and $\eta$ are extreme examples of token reflexivity. Tokens of different token-reflexive expressions can corefer, though there is still no guarantee that synonymous expressions will always corefer. Distinct tokens of "I" and "je", for instance, do corefer when they are used by the same person. But the view we arrived at in the previous section is easily adaptable to them too. Here are the meaning-forms that they share, built out of each of the formulations above:

Character Rule Form: The meaning of $\mathrm{T}$ is the following rule: every token of $\mathrm{T}$ refers to the speaker of that token of $\mathrm{T}$.

Character Function Form: The meaning of $\mathrm{T}$ is the function from each (possible) token of $\mathrm{T}$ to the speaker of that token of $\mathrm{T}$.

Context Function Form: The meaning of $\mathrm{T}$ is the function from each context in which $\mathrm{T}$ is tokened to the speaker of that context.

Truth-Conditional Meaning Form: Any token of a sentence of the form $\phi(T)$ is true just in case $\phi(x)$ is true of the speaker of that token of $T$.

There is a simpler way to state the basic idea here: "I" and "je" play the same role in their respective languages, namely they express the first person singular pronoun. To know the meaning of one is to know a way to express that kind of pronoun (though speakers need not know this fact, since such semantic sophistication is not required for knowing the meaning of "I"). And to know that they are synonymous is to know that they both play the same role, namely that each of their tokens picks out its speaker. 


\section{A Response to an Objection}

In the next section, I will consider possible (and actual) rival proposals. But before that, I need to address a simple objection: meaning-forms are abstractions from meanings; if sameness of an abstraction from meanings is sufficient for synonymy, why not other abstractions? ${ }^{14}$ For instance, the following is an abstraction from the Character Rule Form for "I":

Character Rule Form Abstraction: The meaning of $T$ is the following rule: every token of $T$ refers to something.

If sameness of Character Rule Form Abstraction were sufficient for synonymy, "today" and "I" would count as synonymous. The challenge, then, is to explain why abstracting away the expression is as far as abstraction should go, and, thus, why we cannot also abstract away other features of meaning.

There are two parts to this challenge. The most obvious one can be posed as a slippery slope problem: if we start abstracting some features of meaning, why not others? The second part is one of motivation: is there any good reason to abstract away the expression from the meaning of some expressions, or is it merely an ad hoc way to regain some semblance of synonymy for token-reflexive expressions?

I will begin with the second part, because it will help answer the first part. Token-reflexive meanings differ from non-reflexive ones because their formulation mentions the expression whose meanings they are. If synonymy is to have anything to do with translation between two different languages, there had better be a way to look past the expression itself, and find something that two expressions have in common. And "I" and "je" have everything semantically relevant in common, except their identity. For token-reflexives, then, abstracting away the expression itself is just the right way to get at a sharable meaning.

Which brings us back to the slippery slope problem. Nobody, I assume, would deny that "I" and "today" have some aspect of meaning in common. If all their tokens are indeed guaranteed

\footnotetext{
${ }^{14}$ Thanks to Fiona Doherty for posing this problem.
} 
to refer to something, then they have that in common. ${ }^{15}$ But this sameness does not warrant their being good default translations of each other, nor does learning what one means, and then being told that the other is just like it, suffice for knowing what the other means. I claim, then, that this slope is not all that slippery, and that the abstracting process yields no hint synonymy at other levels than the one I proposed.

One point I do need to grant to this objection: while I have arguments why we are not led by my proposal to thinking that "I" and "today" are synonyms, I have no arguments for the claim that this is the only notion of synonymy other than the traditional one. I see this as an advantage. If there turn out to be other kinds of expressions whose meanings pose other synonymy-related problems, it might turn out that some other kinds of abstraction would be good guides to synonymy.

\section{Other Proposed Solutions}

As with any inconsistent triad, there are several ways to solve the problem of synonymy between token-reflexive expressions. In this section, I will discuss other proposed solutions. Since the issue has mainly arisen in discussions of indexicals, most purported solutions are meant to apply to them. I think of token-reflexivity as a unified phenomenon, and thus will demand of any theory that it count $\zeta$ and $\eta$ as synonymous.

\subsection{No Synonymy}

One option is to reject (C), and claim that there are no synonymous token-reflexive expressions. This will come naturally to someone like Goodman (1949) or Montague (1970, p. 382), who argued that no two expressions are ever synonymous because they are not always intersubstitutable in propositional attitude contexts, or to someone like Quine (1953), who rejected the notion of synonymy altogether. Global skepticism about synonymy is too big an issue for this paper to consider fully. The scope of this paper is quite limited; and, if there are to be any expressions that

\footnotetext{
${ }^{15}$ This would make them automatic indexicals in the sense of Perry (2001).
} 
are synonymous, "I" and "je" would be prime examples (as would $\zeta$ and $\eta$ ). So I leave this reaction aside here.

The other kind of reason to reject (C) depends not on objections to synonymy in general, but on some special features of token-reflexivity. Thus Zimmermann (1997, p. 147), working with a truth-conditional account of meaning:

No two sentences are uttered in precisely the same (possible) situations and hence they cannot have the same truth conditions: the truth conditions of either one are only defined in its contexts of utterance.

The issue raised here is that the meanings of token-reflexive expressions are given in terms of their tokens; but the tokens of one expression are different from the tokens of every other expression, and so we cannot get sameness of meaning.

I have no direct refutation of this view. But I find its conclusion too quick. It is true that meanings, as usually conceived, are too fine grained for finding synonymy between token-reflexive expressions. But meanings are usually conceived this way because philosophers tend to reflect on the meanings of expressions that are not token-reflexive. And for those expressions, there is no difference between meanings and meaning-forms. So I prefer the conclusion that token-reflexivity teaches us something about synonymy, rather than the view that our usual conception of synonymy breaks down when faced with token-reflexivity.

\subsection{Relaxing Token-Reflexivity}

As I said at the beginning, it remains open to say that indexicals are not token-reflexive. If that is correct, my account only applies to artificially introduced expressions like $\zeta$. But one does not need to go that far: one could attempt not to reject, but to modify (A). Let us start with "I" and "je". The problem we are dealing with is that their meanings seem to be about different tokens, since each expression's tokens differ from the other's. So one solution is to make the meanings of these expressions depend not on their tokens, but on any tokens whatsoever. 
One could propose this view simply as a way to solve our original problem. But there is a less ad-hoc way to motivate it, by appealing to certain special features of the first person pronoun, and other context-sensitive expressions, namely that any tokening has someone who counts as the speaker, whether they use the word "I", "je", or, indeed, no pronoun whatsoever. This option would make the meaning of "I" something like the following:

Character Rule for All Tokens: Relative to any token, the meaning of "I" yields the speaker of that token. ${ }^{16}$

Obviously, the right-most part of this rule ("yields the speaker of that token") does not mention "I", and thus the rule will be identical for "je". So we get to preserve sameness of meaning. The cost is that we lose reflexivity, since the rule now yields a referent for the expression relative to all tokens, not just tokens of that particular expression.

I have three objections to this kind of proposal. First, it yields counterintuitive results. Take first $\eta$ and $\zeta$. The most obvious way to adapt the idea to these expressions would be to say that their meaning is the rule which, for every token, yields as referent that very token. But this is not the meaning of $\eta$. In order to know its meaning, one need only know what its tokens refer to. The rest would be baffling to speakers of the language that contains it. Just in case you lack intuitions about $\eta$, think about "I": in order to learn English, you would need to be willing to assign to any token its speaker as a referent.

Admittedly, my proposal has a counterintuitive part too: I am arguing that sameness of meaning is not necessary for synonymy. But this is merely a counterintuitive claim about synonymy. I take my intuitions about the meaning of "I" to be stronger than my intuitions about synonymy. And I would guess that so does the reader.

The second objection is that it predicts that certain questions get a natural answer, even if they do not. Consider this: suppose someone asserts " $2+2=5$ ". It makes sense to ask who said that. But,

\footnotetext{
${ }^{16}$ This is a simplification of the proposal made in $\operatorname{Kupffer}(2001, \S 6.3)$. That account is much more sophisticated (and I should mention that he proposes this as a notion of weak meaning, not full-on meaning, thus also denying (B)), but the objection presented applies to it as well. A similar view is also proposed in Braun (forthcoming), without the accompanying motivation.
} 
putting aside the fact that we have been for so long following Kaplan here, does it make sense to ask "what does 'I' refer to in (or relative to) that assertion?"? My natural response would assume that the person asking that question misheard, and I would just repeat the sentence. As I said above, Kaplan claimed that we need answers to such questions for certain logical and semantic needs. In other work, I argue that these needs are either easily met by a semantics of utterances, or not real needs at all. Here I rest content with the observation that following Kaplan here comes with some costs.

The third objection is that, while it is true that some context sensitive expressions have meanings that depend on contextual features that are independent of the expression being used, not all are. To give an account of the meaning of "I' in this way we rely on the fact that it always makes sense to ask âĂIJwho is the speaker of that token?âĂİ, irrespective of the expression tokened. For demonstratives, like âĂIJthisâĂİ and âĂIJthatâĂİ, we can only ask âĂIJwhat did the speaker intend to refer to by the use of that demonstrative token?âĂ $\dot{I}$, or something similar, and that adverts to a contextual feature that is not independent of the expression used. ${ }^{17}$

The quickest fixes here would be to propose a rule in conditional form, which we could think of as a partial rule, like a partial function. It might look like this:

Conditional Character Rule for All Tokens: Relative to any token, the meaning of "this" yields the demonstratum of / relative to the token, if there is one.

But when does a token have a demonstratum associated with it? Most obviously, when a demonstrative is used. So this rule boils down to a rule that says that "this" plays a demonstrative role in English. But now we are, more or less, where my proposal is: we are thinking of meaning as assigning a role in a language.

\footnotetext{
${ }^{17}$ For more details, see Radulescu (2017).
} 


\section{Conclusion}

In this paper, I proposed a notion of synonymy that can account for token-reflexive expressions: it claims that synonymous expressions share meaning-forms. It is, admittedly, a departure from the customary notion of sameness of meaning, but the difference only really matters when reflexivity comes on the stage, since meaning-forms are identical with the corresponding meanings in all other cases. It is, therefore, a reasonably conservative extension of the customary notion.

I have also argued that other proposals do not account for $\zeta$ and $\eta$, which I take to be just as clearly synonymous as "I" and "je", if not more, since I had the privilege to define their meanings into existence. So, even if it turns out, as I think it will not, that there are no natural language token-reflexive expressions, the notion I proposed still has its uses. But more importantly, I find the other theses, $(\mathrm{A})$ and $(\mathrm{C})$, much harder to give up than accepting $\left(\mathrm{B}^{*}\right)$.

\section{References}

Bach, K. (1992). Intentions and Demonstrations. Analysis, 52, 140-146.

Braun, D. (1995). What Is Character? Journal of Philosophical Logic, 24, 227-240.

Braun, D. (forthcoming). Utterances and Expressions in Semantics and Logic. In K. Turner, \& L. Horn (Eds.) An Atlas of Meaning. Leiden: Brill.

Burge, T. (1978). Self-Reference and Translation. In F. Guenthner, \& M. Guenthner-Reutter (Eds.) Meaning and Translation, (pp. 137-156). New York University Press.

García-Carpintero, M. (1998). Indexicals as Token-Reflexives. Mind, 107(427), 529-563.

Goodman, N. (1949). On Likeness of Meaning. Analysis, 10(1), 1-7.

Hart, W. (1970). On Self-Reference. Philosophical Review, 79(4), 523-528. 
Kaplan, D. (1989). Demonstratives. In J. Almog, J. Perry, \& H. Wettstein (Eds.) Themes from Kaplan, (pp. 481-563). Oxford: Oxford University Press.

Kaplan, D. (1999). Reichenbach's Elements of Symbolic Logic. German translation in Maria Reichenbach and Andreas Kamlah (eds.), Hans Reichenbach: Gesammelte Werke, vol. VI. Frieder: Vieweg.

King, J. C. (2014). Speaker Intentions in Context. Nô̂s, 48(2), 219-237.

Kupffer, M. (2001). Learning French from a Dictionary: An Essay on Utterance-Dependent Meaning. In C. Fery, \& W. Sternefeld (Eds.) Audiatur vox sapientiae: a Festschrift for Arnim von Stechow, Studia grammatica 52, (pp. 320-347). Berlin: De Gruyter.

Montague, R. (1970). Universal Grammar. Theoria, 36(3), 373-398.

Neale, S. (2016). Silent Reference. In G. Ostertag (Ed.) Meanings and Other Things: Themes from the Work of Stephen Schiffer, (pp. 229-342). OUP.

Perry, J. (1997). Indexicals and Demonstratives. In B. Hale, \& C. Wright (Eds.) Companion to the Philosophy of Language, (pp. 586-612). Blackwell.

Perry, J. (2001). Reference and Reflexivity. Stanford University: CSLI.

Predelli, S. (1998). Utterance, Interpretation and the Logic of Indexicals. Mind \& Language, 13(3), 400-414.

Predelli, S. (2004). Think Before You Speak: Utterances and the Logic of Indexicals. Argumentation, 18, 445-463.

Predelli, S. (2006). The Problem with Token-Reflexivity. Synthese, 148, 5-29.

Quine, W. V. O. (1953). Two Dogmas of Empiricism. In From a Logical Point of View, (pp. 21-46). Cambridge, MA: Harvard University Press. 
Radulescu, A. (2017). The Difference between Indexicals and Demonstratives. Synthese, (pp. 1-24). Https://doi.org/10.1007/s11229-017-1367-2.

Reichenbach, H. (1947). Elements of Symbolic Logic. New York: Macmillan.

Schiffer, S. (2003). The Things We Mean. OUP.

Strawson, P. F. (1950). On Referring. Mind, 59(235), 320-344.

Tsohatzidis, S. L. (1992). Pronouns of Address and Truth Conditions. Linguistics, 30, 569-575.

Zimmermann, T. E. (1997). The Addressing Puzzle. In W. Kunne, A. Newen, \& M. Anduschus (Eds.) Direct Reference, Indexicality and Propositional Attitudes, (pp. 133-153). CSLI. 